PHYSICAL REVIEW D 93, 109902(E) (2016)

\title{
Erratum: Spin-dependent two-body interactions from gravitational self-force computations \\ [Phys. Rev. D 92, 124058 (2015)]
}

Donato Bini, Thibault Damour, and Andrea Geralico

(Received 3 May 2016; published 17 May 2016)

DOI: 10.1103/PhysRevD.93.109902

While all of our final results concerning the EOB potentials $\delta A(u, \hat{a})$ and $\delta G_{S}(u, \hat{a})$ (and their decompositions in powers of $\hat{a}$ ) were correct (and correctly printed), some misprints crept in the 8.5PN-level coefficients of the intermediate quantities $-h_{k k}^{(1)}$ and $-\delta U^{(1)}$, as displayed in Eqs. (3.7) and (3.11) of the paper. We list below the corrected values of these (a-linear) 8.5PN-level coefficients.

(1) The last term of Eq. (3.7) should read

$$
\begin{aligned}
-h_{k k}^{(1)}= & \ldots \\
& +\left[-\frac{3268339807}{155925} \ln (u)+\frac{43808}{45} \ln (u)^{2}-\frac{63064680978612989}{922078080000}+\frac{175232}{45} \gamma \ln (u)\right. \\
& +\frac{12252544}{1575} \ln (2) \ln (u)+\frac{70090353336469}{176947200} \pi^{2}-\frac{75337409381}{25165824} \pi^{4}-\frac{923862722}{22275} \gamma-\frac{19712}{3} \zeta(3) \\
& -\frac{102426992006}{1819125} \ln (2)+\frac{175232}{45} \gamma^{2}+\frac{24491392}{1575} \ln (2)^{2}+\frac{24505088}{1575} \gamma \ln (2) \\
& \left.-\frac{34368219}{3080} \ln (3)-\frac{1953125}{792} \ln (5)\right] u^{19 / 2}+O\left(u^{10} \ln u\right) .
\end{aligned}
$$

The last term in the first equation of Eqs. (3.11) should read

$$
\begin{aligned}
-\delta U^{(1)}= & \ldots \\
& +\left[-\frac{2167536532386661}{2521307250}+\frac{780002666754601}{7431782400} \pi^{2}-\frac{40237200436}{16372125} \ln (y)\right. \\
& -\frac{70924306472}{16372125} \gamma-\frac{144895599176}{16372125} \ln (2)-\frac{43593199495}{16777216} \pi^{4}+\frac{10841769}{6160} \ln (3)+\frac{2620096}{1575} \ln (2) \ln (y) \\
& +\frac{437824}{525} \gamma \ln (y)+\frac{5240192}{1575} \gamma \ln (2)-\frac{5504}{5} \zeta(3)-\frac{9765625}{14256} \ln (5)+\frac{109456}{525} \ln (y)^{2} \\
& \left.+\frac{437824}{525} \gamma^{2}+\frac{1744448}{525} \ln (2)^{2}\right] y^{19 / 2} .
\end{aligned}
$$

The resulting expression for $\delta U$ is then in full agreement with the results of Ref. [1] [see Eq. (4.2) there].

(2) At the end of the term between square brackets in the line after Eq. (3.10), one should add a parenthesis ")".

(3) In the first line after Eqs. (3.11), one should replace $O\left(u^{10} \ln u\right)$ by $O\left(y^{10} \ln y\right)$.

(4) Three lines after Eqs. (3.11), $\hat{y}^{13 / 2}$ should be replaced by $\hat{a} y^{13 / 2}$.

We are grateful to B. Wardell for pointing out the misprint in Eq. (3.11).

S. Hopper, C. Kavanagh, and A. C. Ottewill, Analytic self-force calculations in the post-Newtonian regime: Eccentric orbits on a Schwarzschild background, Phys. Rev. D 93, 044010 (2016). 